\title{
Die Auferstehung von Indizien Ermitteln und Erzählen in Friedrich Schillers Geisterseher
}

"Es ist schade, dass man solche Vorfälle nicht genau untersucht, und dass man bei Beurteilung der Begebenheiten [...] immer zwischen verschiedenen Wahrscheinlichkeiten schwanken muss «. ${ }^{1}$ Diese Bedenken zum Verhältnis von Ermittlung und Erkenntnis, von Wahrheit und Wahrscheinlichkeit, die hier Karl in Goethes Unterhaltungen formuliert, teilt Friedrich Schillers Roman Der Geisterseher (1787-1789). ${ }^{2}$ Die im Folgenden vorgeschlagene Lektüre einer unabschließbaren, asymptotischen Indizienlese als Struktur des Fragment gebliebenen Textes legt diesen Schluss in anthropologischer, darstellungstektonischer und wissenspoetologischer Hinsicht nahe.

Zentral ist dabei eine "unglaublich" scheinende "Begebenheit" voller kriminaler, ja "politischer« Vorfälle (48), deren inhärente Semiosekultur Schillers Erzählduktus als unabschließbares Schließen bestimmt - ein Schwanken zwischen Wahrscheinlichkeiten. Diese narrativ unendliche Annährung (Asymptote) rekurriert auf die um 1800 intrikate Ersetzung der einen Wahrheit durch Wahrscheinlichkeiten: Das Verhältnis des Zeichens zu seiner "Repräsentationskraft stürzt in sich zusammen «. ${ }^{3}$ Diese Bestandsaufnahme unterzieht Schiller in Der Geisterseher einer literarischen Prüfung, in der er das instabilisierte Verhältnis zwischen res und verba als einen, wie er Goethe gegenüber bekennt, "Abgrund « empfindet, »in den ich nicht ohne Schwindeln schauen kann «, ${ }^{4}$ den er aber nicht nur auslotet, sondern, so möchte ich zeigen, radikal vertieft.

Den Zusammenhängen zwischen verdächtigen Tatsachen und zu ziehenden Schlüssen, Ermitteln und Erzählen im Geisterseher werde ich in drei Schrit-

1 Johann Wolfgang v. Goethe: Unterhaltungen Deutscher Ausgewanderten, in: Sämtliche Werke, Münchner Ausgabe, Bd. 4/1, hg. v. Rainer Wild, München 1988, S. 469.

2 Friedrich Schiller: Der Geisterseher, in: Sämtliche Werke. Erzählungen, Theoretische Schriften, Bd. 5, hg. von Wolfgang Riedel, München 2004, S. 48-182. Zitate aus Der Geisterseher werden im Folgenden direkt im Text in runden Klammern ausgewiesen.

3 Wolfgang Schäffner: Medialität der Zeichen, in: Das Laokoon Paradigma. Zeichenregime im 18. Jahrhundert, hg. von Inge Baxmann und Michael Franz, Berlin 2000, S. 274-290, hier S. 277.

4 Brief von Schiller an Goethe vom 27. Februar 1798, in: Schiller, Werke und Briefe in zwölf Bänden, Bd. 11: Briefe II, hg. von Georg Kurscheidt, Frankfurt a.M. 2002, S. 377. 
ten nachgehen: Erstens werde ich über eine kurze Rekapitulation der relevanten Stationen des Geistersehers vor Augen stellen, warum die verschwörungstheoretische Anlage des Romans eine asymptotische Bewegung der Aufklärung und Verunklarung mittels Indizien favorisiert. Im zweiten Teil werde ich eine historische Einordnung vornehmen, die sich auf Indizien im juridischen Diskurs des ausgehenden 18. Jahrhunderts als Entstehungshorizont des Romans bezieht. Indizien werden dabei als Ermittlungspraxis und ebenso vielversprechendes wie umstrittenes Rechtsinstrument konturiert. Drittens werde ich anhand von ausgesuchten Lektürevignetten verfolgen, wie Schillers Text als ermittelndes Erzählen agiert: vom Etablieren einer affirmativen Indizienlese über die erkenntnistheoretische Absage an Indizien bis hin zu ihrem narrativen Wiedereinsatz als asymptotisches Erzählverfahren - ein Umstand, der Indizien auf der doppelten Ebene von Dargestelltem und Darstellung poetologisch unverzichtbar macht.

\section{Geister sehen und Geister rufen}

Der Geisterseher ist ein Gattungsamalgam aus Schauergeschichte, Briefroman, philosophischem Traktat, Memoiren, theatralen Dialogen und Detektiverzählung, das ab 1787 (bis 1789) als Fortsetzungsroman in sechs Lieferungen in Schillers Zeitschrift Thalia erscheint. Der Text greift zeitgenössische Motive und Diskurse der Aufklärung am Vorabend der französischen Revolution auf und zeichnet das ausgehende Siècle des Lumières als desaströs aufgeladene Übergangszeit zwischen Überdruss an der alten (kartesianischen) und voller Misstrauen gegenüber einer zukünftigen (romantischen) Ordnung: Die "metaphysische Anämie ${ }^{5}$ der Aufklärung evoziert einen geradezu trostlosen Bedarf an Wunderglauben, Magie und Übernatürlichem, die in die entstandene Transzendentallücke einrücken sollen. Diese tiefe Verunsicherung findet sich literarisch gespiegelt in der formalen Disparatheit des Geistersehers als Genrekollision, Tiefenschachtelung der Erzählebenen, Unzuverlässigkeit der Erzählerinstanz, die zudem ständig wechselt, und nicht zuletzt intradiegetisch in der Figur eines sympathischen, aber schwachen,

5 Hans Richard Brittnacher: Dunkelmänner im Licht. Dialektik der Aufklärung in Schillers Geisterseher, in: Übersetzen, Übertragen, Überreden, hg. von Sabine Eickenrodt, Stephan Porombka und Susanne Scharnowski, Würzburg 1999, S. 173-183, hier S. 175. 
stets zwischen intellektueller Ermächtigung und Schwärmerei, Altruismus und Geltungssucht schwankenden Prinzen.

Die anhaltende Begeisterung, die der Text seit seiner sukzessiven Entstehung bei seinem Publikum trotz (oder wegen) seines fragmentarischen Charakters auslöst, teilt sein Autor keineswegs: Schiller hält den "verfluchten Geisterseher« für »sündlichen Zeitaufwand« und ist überzeugt, »diese Schmiererei« werde durchweg "schlecht - schlecht«. Gleichwohl trifft er den "Geschmack des Publikums" so souverän, dass er sich vornimmt, wenigstens "so viel Geld davon [zu] ziehen, als nur immer möglich ist «. ${ }^{6}$ Die vehemente Forderung nach Fortsetzung seitens der Leserschaft hängt nicht zuletzt mit der, vor allem in den ersten beiden Lieferungen kolportageartig gehaltenen "Darstellung von Magie, Hellseherei, dynastischen Intrigen, Geheimbünden und Verschwörungen" zusammen, die auf die "Nachtseite der Aufklärung « gehören. ${ }^{7}$ Gegen die vernunftzentrierten Werte von Wahrheit, Toleranz und Sittlichkeit hält der Text die erklärten Feindbilder der Aufklärung wie Schwärmerei, Ignoranz, Betrug, Sinnlichkeit und Fremdbestimmung. Die prozessuale Verhandlung beider Seiten ist im Roman geschickt als perpetuum mobile inszeniert, so dass oft genug scheinbare Widersprüche in ein Ersetzungsverhältnis umschlagen, wenn beispielsweise wachsame Aufmerksamkeit in Verfolgungswahn oder hingebungsvolle Dienstbarkeit in Überwachung umschlagen.

Das Erzählen präsentiert sich in diesem janusköpfigen Arrangement als Ermitteln, als Modus der unnachgiebigen Aufklärung über eine Sache oder als objektiv auftretende Vermittlung von Wissen. Diese bohrende Richtung des Erzählens als Ermitteln wechselt jedoch im Fortgang der Handlung immer wieder über von der Aufklärung in eine Verdunklung der Zusammenhänge - nicht umsonst bildet das Handlungssetting der maskenreiche Karneval in Venedig. Der perpetuierte Modus der erzählenden Ermittlung führt im Roman dazu, dass hinter jedem zunächst aufgeklärt scheinenden Zusammenhang bereits die nächste Maske der Verrätselung auftaucht, hinter der wiederum der nächste Verdacht keimt: So mutiert das erzählende Ermitteln zu einem verschwörungstheoretischen Erzählmodell ohne Stoppregel. ${ }^{8}$ Der

6 Schillers kritische Selbsteinschätzungen finden sich beispielsweise in Briefen von Schiller an Göschen vom 3.3.1787 und an Körner vom 17.3.1788 und 15.5.1788, hier zit. nach dem Kommentar von Wolfgang Riedel zu Friedrich Schiller, Der Geisterseher, S. 1160.

7 Dirk Oschmann: Friedrich Schiller, Stuttgart 2011, S. 71.

8 Vgl. zu diesem eskalativen Zusammenhang und der darin produzierten "interpretatorischen Gefräßigkeit« Ralf Simon: Commercium und Verschwörungstheorie. Schillers Geisterseher und Jean Pauls Titan, in: Jahrbuch der Jean-Paul-Gesellschaft 41 (2006), 
Modus eineindeutiger Aufklärung verfällt der Heteronomie asymptotischer Viel- und Weiterdeutung und entfesselt eine interpretatorische Eskalation der (Un-)Wahrscheinlichkeiten.

Ein kurzer Blick auf die Handlung soll zunächst die Orientierung in der konspirativen Schachtelung des Textes erleichtern: Erzählt wird die Geschichte eines protestantischen Prinzen, der trotz "strengsten Inkognitos" (48) während seines Aufenthalts in Venedig Opfer einer kunstvoll gesponnenen Intrige wird, die sich mit unheimlichen Prophezeiungen, rätselhaften Todesfällen, Geisterbeschwörungen, Liebesabenteuer, Glücksspiel, finanziellem Ruin und der verheißungsvollen Möglichkeit zur Thronfolge verbindet. Zurückzuführen sind all diese den Prinzen einspinnenden, manipulativen und skrupellosen Machenschaften auf eine jesuitische Geheimgesellschaft, deren genialer Kopf der "Armenier«, ein Illusionist vom Format Cagliostros ist. ${ }^{9}$ Das Ziel der konfessionell motivierten und damit machtpolitisch ausgerichteten Intrige ist es, den Prinzen innerlich so zu zerrütten, emotional so gefügig und finanziell so abhängig zu machen, dass er zum Katholizismus konvertiert und sich durch den mörderischen Komplott widerrechtlich der Krone seiner Heimatprovinz bemächtigt, die so für den Machteinfluss der Jesuiten gewonnen wäre. Der Text bricht jedoch mit der insinuierten Konversion des Prinzen ab, der in den "Armen« des Armeniers »die erste Messe hörte« (160). Von der machtpolitischen Usurpation wird nicht mehr explizit berichtet, nur der Anfang des Romans verspricht den "Schlüssel« $\mathrm{zu}$ »einem gewissen politischen Vorfalle» (48) zu liefern, dieser geht aber in

S. 221-245, hier S. 221; thematisch grundlegend zum Zusammenhang von Aufklärung und Verschwörungstheorie vgl. Ralf Klausnitzer: "... unter allen möglichen Gestalten und Konnexionen«. Die Geburt des modernen Konspirationismus aus dem Geist der Aufklärung, in: Zeitschrift für Geschichte 11/3 (2004), S. 13-35.

$9 \mathrm{Zu}$ historischen Hintergründen und ihrer poetischen Motivaufnahme sowie poetologischen Wirkung im Geisterseher von Freimaurertum über Spiritismus bis Gegenaufklärung äußert sich eine Reihe von einschlägiger Forschungsliteratur: Klaus H. Kiefer: »Die famose Hexenepoche«. Sichtbares und Unsichtbares in der Aufklärung, München 2004; Matthias Hurst: Im Spannungsfeld der Aufklärung. Von Schillers Geisterseher bis zur TV-Serie The X-Files: Rationalismus und Irrationalismus in Literatur, Film und Fernsehen 1786-1999, Heidelberg 2001; besonders instruktiv zum reziproken Verhältnis von Aufklärung und Gegenaufklärung argumentieren: Hans Richard Brittnacher: Dunkelmänner im Licht; Monika Schmitz-Emans: Zwischen wahrem und falschem Zauber. Magie und Illusionistik als metapoetische Gleichnisse, in: ZDP 115 (1996), Sonderheft: Klassik, modern, S. 33-43; Liliane Weisberg: Geistersprache. Philosophischer und literarischer Diskurs im späten 18. Jahrhundert, Würzburg 1990; vor allem historisch quellenreich arbeitet Michael Voges: Aufklärung und Geheimnis. Untersuchungen zur Vermittlung von Literatur- und Sozialgeschichte am Beispiel der Aneignung des Geheimbundmaterials im Roman des späten 18. Jahrhunderts, Tübingen 1987. 
der narrativen Lücke zwischen Ankündigung und ausbleibendem Finale des Romantorsos verloren.

Wenn aber ein Text als »Memoires« damit einsetzt, über eine »Begebenheit, die vielen unglaublich scheinen wird«, mit einem Ich-Erzähler als »Augenzeuge« dem Leser »willkommenen Aufschluß« zu liefern, ja ihm den maßgeblichen "Schlüssel« für diese "Geschichte des Betrugs" an die Hand zu geben verspricht, wird narrativ eine Ermittlungslogik und mit ihr juridische Topoi aufgerufen, die dazu angetan sind, auf ihren erzählstrategischen Einsatz im Namen der postulierten »reinen, strengen Wahrheit» (48) hin befragt zu werden. Diese Topoi sind um 1800 in den rechtswissenschaftlichen Debatten des diskursiven Entstehungshorizonts von Schillers Geisterseher einschlägig und gut zu identifizieren. Denn strafprozessrechtlich werden hier über den Romantext zwei zeitgenössisch konkurrierende Rechtsinstrumente bei der Ermittlung (»Wahrheit«) eines kriminalen Geschehens (»Vorfalle«) aufgerufen: erstens die Zeugenschaft (»Augenzeuge«, "Memoires«) und zweitens das Indiz (»Aufschluß«, "Schlüssel«). Zum Verständnis der Genese dieser Konkurrenz sowie der jeweiligen historischen Deutungshoheit beider Rechtsinstrumente soll der folgende Exkurs den argumentativen Boden bereiten. Erst auf der rechtshistorischen Basis lässt sich zeigen, dass den Indizien, so meine These, im Geisterseher eine diskursiv wie metapoetisch überlegene Wirkmächtigkeit gegenüber der verdächtig gewordenen Qualität von Zeugenschaft, respektive Geständnis, zugesprochen wird.

\section{Ermittelndes Erzählen}

Kein tatsächliches Gerichtsverfahren bildet im Geisterseher intradiegetisch die Ermittlungspraxis ab, ${ }^{10}$ sondern übergeordnet das Erzählverfahren selbst. Genretektonisch und damit formal gestützt wird diese Konstitution über die erste und zweite Lieferung, die der Logik von Rätsel (Geistererscheinung) und Auflösung (Prinz als Ermittler) folgen: Die Lesenden werden nach ei-

10 Eine gewisse, im inquisitorischen Vorgehen radikale und "Entsetzen« (54) auslösende Ausnahme bildet das Standgericht der venezianischen "Staatsinquisition", die einen dem Prinzen nach dem Leben trachtenden, so die Anklage, rachsüchtigen "Venezianer" (53) - der Prinz hatte ihn beim Glücksspiel vermeintlich um den sicher geglaubten Gewinn gebracht und ihm die Stirn geboten - nach allerkürzester Inquisition und unmittelbarem Schuldeingeständnis noch vor den Augen des Prinzen enthauptet, so dass »der Gerechtigkeit in Venedig« genüge getan ist, der Prinz aber darob "ohnmächtig« (54) niedersinkt. 
nem veritablen Cliffhanger am Ende des ersten Teils dann im zweiten Teil an die kriminalistisch geschult wirkende Hand genommen, wobei der Prinz zum Helden einer Detektiverzählung avant la lettre avanciert. Bevor ich im übernächsten Schritt die unerklärlich scheinenden Phänomene und ihre indiziengeleitete Aufklärung seitens des Prinzen rekapituliere, sollen die von Schiller im Geisterseher so opulent verteilten gegenständlichen Hinweise wie Ehering, Taschenuhr oder (indizienlogisch sinnfällig) ein Schlüssel in das sie als Indiz qualifizierende Paradigma des Denkschlusses eingeordnet und damit als Grenzgänger im Spannungsfeld von Tatsache und semantischer Ordnung positioniert werden.

\section{II.1 Indizien um 1800}

Neue Ermittlungstechniken identifizieren im 18. Jahrhundert Gewalt als dysfunktional zum Zwecke der Informationsgewinnung. Exemplarisch für die Variabilität und Funktionalisierung der Kontrolle ist die Bewegung von der unmittelbaren Informationsgewinnung (Folter) zum modernen Beobachtungs- und Distanzparadigma. Die von Foucault beschriebene Verinnerlichung ${ }^{11}$ der $»$ Disziplinarmacht ${ }^{12}$ charakterisiert die Strafrechtspolitik im 18. Jahrhundert. Die Abschaffung der Folter, die im 18. Jahrhundert die Diskussion über neue Rechtsinstrumente initiiert, veranschaulicht die Verlagerung von direkt exekutierter Gesetzesgewalt am Körper des Delinquenten hin zu variableren Realitäts- und Wahrheitsprozeduren der reformierten Kriminalordnungen, die sich als spezifisch mittelbare Ermittlungspraxis über den Denkschluss beschreiben lassen, der Tatsachen zu einem Sachverhalt oder Tathergang zusammenschließt.

Im Zuge der Rechtsreformen um 1800 lässt sich eine allmähliche strafrechtliche Verschiebung beobachten: vom geständigen Täter oder Zeugen hin zu ermittlungstechnisch praktikableren und in der Aufklärung von Verbrechen effizienteren Indizien, die allerdings nur für eine gewisse »Wahrscheinlichkeit« einzustehen vermögen. ${ }^{13}$ Rechtshistorisch lässt sich dieser (bis

11 Vgl. zur These der Internalisierung der Disziplinarmacht Michel Foucault: Überwachen und Strafen. Die Geburt des Gefängnisses, Frankfurt a. M. 2008, sowie ders.: Dispositive der Macht. Über Sexualität, Wissen und Wahrheit, Berlin 1978, und ders.: Analytik der Macht, Frankfurt a.M. 2005, S. 126-174.

12 Ders.: Analytik der Macht, S. 148ff., so auch: Ders.: Dits et Ecrits III, Nr. 239: ’Die Gouvernementalität<, Frankfurt a.M. 2005, S. 820.

13 René Pöltl: Die Lehre vom Indizienbeweis im 19. Jahrhundert, Frankfurt a.M. 1999, S. 142. 
heute geltende) Umstand folgendermaßen herleiten: In der frühneuzeitlichen Constitutio Criminalis Carolina (1532) ${ }^{14}$ dem ersten deutschen Strafrecht überhaupt, können Indizien noch nicht zur Verurteilung führen. Die als reformistische Großtat geltende Carolina führt Indizien (»redlich anzeygung«, $C C C, \S 18)$ in den Inquisitionsprozess zwar ein, allerdings nur, um die Anwendung der Folter zu regeln. Ausschlaggebend für ein rechtskräftiges Urteil sind das (erfolterte) Geständnis des Täters oder die Aussage zweier Zeugen mit tadellosem Leumund: "Genugsame Zeugen« aber "seindt die, die vnverleumbdt vnnd sunst mit keiner Rechtmessigen vrsach zuwerffen seindt" $(C C C, \S 66)$ - diese Regelung erwies sich in den Strafprozessen als nicht praktikabel und trug zur Aufwertung von Indizien innerhalb der Jurisprudenz bei. ${ }^{15}$

Nach der Abschaffung der Folter klafft so in der Strafprozessrealität eine Rechtslücke und es steht dringend eine Einigung über die Rechtsnatur von Indizien an. Diese Debatte lässt sich im ausgehenden 18. Jahrhundert an einem jahrzehntelangen Schlagabtausch verfolgen, der zwischen Befürwortern der Indizien, die deren ermittlungstechnisch akkumulierbare Objektivität preisen, und deren Gegnern, die die Fehleranfälligkeit dieses sentsubjektivierten Verhandelns von Wahrscheinlichkeiten bemängeln, entbrennt. Erst Mitte des 19. Jahrhunderts konstituiert sich eine dauerhafte Lösung, die beiden Seiten Rechnung trägt, da zwei Beweissysteme miteinander kombiniert werden: Das verfügbare Material (Indiz) wird einem rechtskundigen, erfahrenen, das Material als relevant oder irrelevant qualifizierenden (Liminalität von Indizien) und ausdeutenden (Heteronomie von Indizien) Subjekt (Richter) für die sogenannte freie Beweiswürdigung vorgelegt. ${ }^{16}$

14 Die maßgeblich von dem reformorientierten Richter Johann Freiherr von Schwarzenberg konzipierte Constitutio Criminalis Carolina (im Folgenden abgekürzt als CCC), die Peinliche Halsgerichtsordnung Karls V., trat 1532 als das erste allgemeine deutsche Strafgesetzbuch in Kraft.

15 „Doch die Tendenz, das Geständnis gegebenenfalls durch physische Gewalt zu erpressen, hing aufs engste mit der gesetzlichen Beweisregelung zusammen, wonach das Gericht die zur Verurteilung hinreichenden Tatsachen nur dann als erwiesen (facta plene probata) ansehen durfte, wenn zwei vollgültige Zeugen sie bekundeten (das war sehr selten) oder wenn der Inquisitus gestand.«, Eberhard Schmidt: Einführung in die Geschichte der deutschen Strafrechtpflege, Göttingen 1995, S. 270.

16 In Preußen tritt diese bis heute geltende Regelung 1846 und in der Reichsstrafprozessordnung 1877 in Kraft, vgl. Rudolf Stichweh: Zur Subjektivierung der Entscheidungsfindung im deutschen Strafprozess des 19. Jahrhunderts. Aspekte der Ausdifferenzierung des Rechtssystems, in: Subjektivierung des justiziellen Beweisverfahrens, hg. von André Gouron, Frankfurt a.M. 1994, S. 265-300; hierzu auch René Pöltl: Indizienbeweis, S. 471. 
Unter Indizien fällt bereits um 1800 alles, was auf den strafrechtlich relevanten Zusammenhang schließen lässt. Dies beschreibt beispielhaft die Preusische Criminalordnung (1805), ${ }^{17}$ wenn sie von »erwiesenen Thatsachen« spricht, dank derer auf das Verbrechen, dessen Ursache, dessen Bedingung oder dessen Urheber geschlossen werden kann:

Dies [ihre Wahrscheinlichkeit; A.E.] gilt von erwiesenen Thatsachen, die entweder eine bestimmte Bedingung oder Ursache des Verbrechens in sich enthalten, oder das Verbrechen als Ursache oder Bedingung voraussetzen, und woraus daher auf das Verbrechen oder dessen Urheber geschlossen werden kann. (CO, § 399; Hervorh. A.E. $)^{18}$

Die Begriffe Thatsache und Denkschluss formieren damit die zentralen Angelpunkte einer Argumentation, die auf zeitlich rückwärts gerichtete (retrospektiv) ebenso wie auf eine ins Zukünftige zielende (prospektive) Kombinatorik als Ermittlungsperspektive rekurrieren.

Der Begriff Tatsache hat im Deutschen eine überraschend junge Geschichte. ${ }^{19}$ Das Wort ist eine Neubildung des 18. Jahrhunderts, die vom juridischen res facti übernommen bald in die philosophische und philologische Diskussion gerät. ${ }^{20}$ Thatsachen werden um 1800 nicht ausschließlich im Sinne eines Faktums gebraucht, sondern als Terminus, der einerseits bereits "menschliche Taten« bezeichnet und andererseits "evidentielle Darstellung bzw. Vergegenwärtigung in Form von Erzählungen « postuliert. ${ }^{21}$ Solche Tat- und Handlungs-Sachen sind also im Sprachgebrauch um 1800 nicht

17 Preußische Criminalordnung, Berlin, 11. Dezember 1805, im Folgenden direkt im Text zitiert als $\mathrm{CO}$ mit nachfolgendem Paragraphen.

18 Die Nähe zwischen der heute geltenden Formulierung über den Begriff des Schließens und §399 der Preußischen Criminalordnung von 1805 ist beträchtlich, wenn es in einer Entscheidung des Bundesgerichtshofs heißt: "Hauptstück des Indizienbeweises ist also nicht die eigentliche Indiztatsache, sondern der daran anknüpfende weitere Denkproze/s, kraft dessen auf das Gegebensein der rechtserheblichen weiteren Tatsache geschlossen wird« (BGHZ 53 [1970], S. 260f.; Hervorh. A.E.).

19 Gezeigt hat das jüngst Johannes Lehmann: Faktum, Anekdote, Gerücht. Zur Begriffsgeschichte der >Thatsacher und Kleists Berliner Abendblättern, in: Deutsche Vierteljahrsschrift für Literaturwissenschaft und Geistesgeschichte 89/3 (2015), S. 307-322. Ich danke Johannes Lehmann für die Einsicht in sein Manuskript. Für den englischsprachigen Kulturkontext einschlägig ist der Begriff "facts" bei Francis Bacon, vgl. Lorraine Daston: Baconsche Tatsachen, in: Rechtsgeschichte 1 (2002), S. 36-55. Dazu auch Barbara Shapiro: A Culture of Fact. England 1550-1720, London 2000, besonders S. 105-138.

20 Vgl. zur Einwanderung des Begriffs aus dem Rechtszusammenhang in die Theologie Reinhart Staats: Der theologiegeschichtliche Hintergrund des Begriffs >Tatsacher, in: Zeitschrift für Theologie und Kirche 70/1 (1973), S. 316-345.

21 Johannes Lehmann: Faktum, Anekdote, Gerücht, S. 310. 
Fakten tout sec, sondern immer schon Handlungen: Tatsachen sind bereits Geschichten, die erzählen, die Geschehen motivieren und evident machen. So steht der Begriff Thatsache in seiner Genese im Ausgang des 18. Jahrhundert bereits am Schnittpunkt zwischen juridischer und narrativer Kultur und damit in unmittelbarer Nachbarschaft zur Diskussion um die Indizien.

\section{II.2 Doppeltes Wissen: Retrospektive \& Prospektive}

An genau dieser Stelle rücken die begrifflich so jungen »Thatsachen" nun in die Preufsische Criminalordnung ein sowie semantisch mit den indizierenden "Tatsachen" (75) aus Schillers Geisterseher zusammen und werden über Schlüsse, retrospektive und prospektive, mit Hergang und/oder Fortsetzung von Handlung verknüpft. In seinem Roman nutzt Schiller, und dies ist für die Ermittlungspraxis und Erzähldynamik symptomatisch, beide Richtungen des Schließens, um das zeitlich Unzugängliche verfügbar zu machen: Retrospektive und Prospektive. Zunächst dominiert im "1. Buch" (48), den ersten beiden Lieferungen des Fortsetzungsromans, die retrospektive Indizienlektüre: Die Geistererscheinungen werden durch die ermittelnde Analyse im dialogischen Gespräch zwischen Prinz und Graf von $\mathrm{O}^{* *}$ rekapitulierend und rekonstruierend aufgeklärt - ein, wie sich zeigen wird, die weitere Entwicklung stimulierender, nicht aufösbarer Rest bleibt jedoch. Über die anschließend im Medium des Briefes evozierte Gegenwärtigkeit erlangt das Erzählverfahren ab der dritten Lieferung, wenn die »Memoires« des Grafen von $\mathrm{O}^{* *}$ durch eine lange Abfolge von zehn Briefen des Baron von $\mathrm{F}^{* * *}$ abgelöst werden, einen iterativen, seriellen und präsentischen Erzählduktus, der die Souveränität des retrospektiven Blicks einbüßt. Den über das Geschehen nun informierenden Briefen ist zudem eine kryptische Vorausdeutung des Grafen vorangestellt, die das fatale Ende der kommenden, katastrophischen Entwicklung, den Niedergang der "so wohl verteidigten Vernunft« und den Erfolg der »unerhörten Teufelei« (104) zwar vorwegnimmt, aber darin ganz unspezifisch in Bezug auf das Wie bleibt. Diese narratologische Anlage evoziert im »2. Buch« (104) eine doppelte Erzähl- und d.h. auch eine doppelte Ermittlungsperspektive: Einerseits nämlich wird über die massive Vorausdeutung seitens des Erzählers ein Wissen-Können behauptet, das nun nicht mehr den detektivischen Prinzen, sondern in einer metaleptischen Strategie die Lesenden selbst zu Ermittlungsleistungen motiviert. Wie das vom Erzähler Annoncierte zur Entfaltung kommt, können die Rezipienten vor diesem Wissenshorizont im Fortgang der Lektüre ermitteln, indem sie die Heteronomie der Indizien (Vieldeutigkeit von Indizien) einhegen und ihre 
Liminalität (Was wird als Indiz qualifiziert?) festlegen. Nicht das Whodunit (fraglos der Armenier), sondern das Wie wird im zweiten Buch fokussiert. Und ebendies führt neben dem (diffus bleibenden) Retrospektivhorizont der Rahmenhandlung (Wissen des Erzählers) andererseits auf der Ebene der Binnengeschichte (Briefe) zu einem prospektiven Vorgehen, einem geradezu entelechisch anmutenden Zurichten und Auslegen von Zeichen durch den Armenier. Die Lesenden stehen dabei genau auf der Schwelle dieser zeitlichen Doppelordnung des Wissens zwischen Retrospektive und Prospektive, indem sie durch das exoterische Generalwissen des Erzählers zwar über den fatalen Ausgang informiert sind, nicht aber esoterisch in die Anlage der jeweiligen Ränkespiele eingeweiht werden. Über diese Konstellation aus gleichzeitigem Wissen und Nichtwissen ergibt sich eine Lesesituation des unsicheren Wissen, das seiner Anlage nach konstitutiv für den Romantorso ist: Der Fragment gebliebene Text generiert gerade keine Wahrheit, sondern Wahrscheinlichkeiten. Wie diese zwischen $\mathrm{zu}$ entschlüsselnden Zeichen und der Manipulation von Erzähltem, Erzähler(n), Figuren und Lesenden oszillieren, zeigt der folgende, letzte Teil meiner Ausführungen. Ebenso resümierend wie vorausschauend sei hier zunächst festgehalten: Im Geisterseher wird indiziert ermittelt und indizierend erzählt.

III. Aufstieg und Niedergang von Indizien

„Wollen Sie lieber Wunder glauben als eine Unwahrscheinlichkeit zugeben?« (97), spottet der Prinz über den Grafen von $\mathrm{O}^{* *}$, indem er das Unwahrscheinliche zum Wahrscheinlichen erklärt und damit zentrale Diskussionen um das Indiz alludiert. Strafrechtliche Theorie und Praxis beruhen am Ende des 18. Jahrhunderts nicht mehr länger auf der Prämisse einer zumindest behaupteten "Gewissheit « in Bezug auf die Urteilsfindung, sondern auf »ungewisse $[\mathrm{r}]$ Erkenntnis « $^{22}$ - und mithin auf Wahrscheinlichkeit. So verhandeln thematisch einschlägige Autoren wie Kleist oder E.T.A. Hoffmann in ihren Texten kritisch die Unsicherheit juridischer Ermittlungsmethoden, die in literarischer Konsequenz noch wesentlich unsicherere Erkenntnisverfahren wie den rgesunden Menschenverstand oder die Intuition bei der Aufklä-

22 Paul Johann Anselm von Feuerbach: Lehrbuch des gemeinen in Deutschland gültigen peinlichen Rechts [1801], 5. Aufl., Gießen 1812, S. 479. 
rung einsetzen - diese Texte favorisieren ein gefühltes Wissen. ${ }^{23}$ Bei diesem Wissenstyp verbinden sich wissenschaftliche Methoden des Vergleichs mit emotionalen Formen des Instinkts, der Phantasie und Erfahrung zu »elastischer Härte«. ${ }^{24}$ Ins Paradigma dieser Ermittlungstechnik gehören zentral die Indizien. Damit qualifiziert sich der indiziengeleitete Denkschluss als eine Form der Ermittlungspraxis, die wissenschaftliches und ästhetisches, rationales und emotionales Wissen kombiniert.

Die Verknüpfung, die nun Schillers Romanpersonal zwischen Tatsachen und Sinnstiftung herstellt, läuft im Geisterseher auffällig oft - und auffällig erfolgreich - über Indizien. Deren Funktionsweise wird zunächst in der affirmativen Objektzuversicht des Aufklärers und einer deutlichen Skepsis gegen das Modell Zeugenschaft ausgestellt (III.1), dann in einem verschwörungslogischen Überschuss aufgesprengt (III.2), um in einem finalen Doppelschritt neu konstituiert (III.3), zugleich aber fundamental und final problematisiert (IV) zu werden.

\section{III.1 Der Sizilianer: Eine verdächtig aufgeklärte Affäre}

Schon die erzählstrategische Anlage des Geistersehers unterminiert traditionelle Konzepte von Glaubwürdigkeit und zwar gezielt sowohl über die für sie einstehende Figur sowie über den exponiert instabilen Überlieferungsstatus der Geschichte. Beispielsweise werden die literarisch sonst mit besonderer Glaubwürdigkeit ausgestatteten Ich-Erzählungen jeweils ausgerechnet von infamen Betrügern erzählt: So die Vergangenheit des Sizilianers, die vor allem der Gestalt des Armeniers geheimnisvolle Tiefe und unverbrüchliche Authentizität verleihen soll, ebenso wie die voyeuristische Episode, die Civitella schildert und in der er Zeuge des Abschieds zwischen der »Grie-

23 Vgl. zur Rolle von Indizien bei Kleist: Antonia Eder: Doing Truth. Indizien und verdächtige Schlüsse bei Heinrich von Kleist, in: Dichterjuristen. Studien zur Poesie des Rechts vom 16. bis 21. Jahrhundert, hg. von Yvonne Nilges, Würzburg 2014, S. 67-89; und zu E.T.A. Hoffmann vgl. Antonia Eder: »Welch dunkles Verhältnis der Dinge«. Indizienlese in E.T.A. Hoffmanns Das Fräulein von Scuderi, in: Spiegelungen - Brechungen. Frankreichbilder in deutschsprachigen Kulturkontexten, hg. von Marion George, Berlin 2011, S. 263-286.

24 Carlo Ginzburg: Spurensicherung. Der Jäger entziffert die Fährte, Sherlock Holmes nimmt die Lupe, Freud liest Morelli - die Wissenschaft auf der Suche nach sich selbst, in: Spurensicherungen. Über verborgene Geschichte, Kunst und soziales Gedächtnis, München 1988, S. 78-124, hier S. 91. 
chin« (136) und ihrem Liebhaber (dem Armenier) wird. ${ }^{25}$ Gerade der Status dieser Episode ist hochgradig verdächtig: Nicht nur dass Civitella diesen wertvollen Hinweis auf Identität und Aufenthaltsort der vom Prinzen angebeteten und längst verzweifelt gesuchten Griechin ausgerechnet »erinnerte« (142), als dessen finanzielle Abhängigkeit von Civitellas Onkel, dem Kardinal, unumkehrbar ist. Die Glaubwürdigkeit der Geschichte wird zudem formal dadurch zersetzt, dass sie als "Ich-Erzählung Civitellas in einem Brief des Baron von $\mathrm{F}^{* * *}$ erscheint, der wiederum von den Memoiren des Grafen von $\mathrm{O}^{* *}$ mitgeteilt wird, die ihrerseits von einem anonymen Herausgeber kommentiert werden «. ${ }^{26}$ Die vierfache Brechung des Editionsweges sowie die verdächtige Verzögerung der Erinnerung des ansonsten aufmerksamen Zeugen Civitella markieren die zu lesenden Zeichen dieser Begebenheit als hochgradig konstruiert und korrumpiert. Anhand solcher Pointen diskreditiert Schillers Roman das juridisch noch geltungsstarke Modell der Zeugenschaft, das für die eine Wahrheit einzustehen vermeint.

Die bereits erwähnte Ich-Erzählung als im Roman ausführlich entfaltete Vorgeschichte des Sizilianers kontrastiert in genau dieser argumentativen Stoßrichtung die Rechtsinstrumente Zeuge und Indizien, wobei ersterer in Person des Ich-Erzählers (»Nichtswürdiger«, 93) schon als defizitär markiert ist und letztere nicht nur zur Aufklärung dubioser Vorgänge, inklusive der Geistererscheinung, beitragen, sondern zum entscheidenden Movens der, wenn auch intrikaten, Romandynamik avancieren.

Nach seiner Verhaftung gibt der Sizilianer beim Verhör bereitwillig die Erklärungen zu den vorangegangenen rätselhaften Ereignissen, die, begünstigt durch seine eigene "Geschwindigkeit«, die "Dämmerung" und das »Erstaunen« des Prinzen, »diesen Betrug« (71) vervollkommnen. So klären sich sukzessive die überraschende Enttarnung des Prinzen durch die vom Sizilianer informierte Tänzerin (73) ebenso wie die vermeintlich schicksalhafte Zulosung des Gewinns einer Dose in der Lotterie, die den verlorenen und vom Sizilianer entwendeten »Schlüssel« (72) birgt, oder die Ähnlichkeit des vom Sizilianer qua "Zauberlaterne« (73) evozierten Geistes mit dem verstorbenen Freund des Prinzen. Das "Wunderbare« (73), das während der Geisterbeschwörung die "Einbildungskraft spann[t] $[$, entpuppt sich als äußerst bodenständige "Maschine«, deren »elektrischer Schlag« per "Kruzifix»

25 Schiller schrieb die Novelle Der Abschied als sechste und letzte Lieferung des Romans; in der Buchfassung wurde sie später in den "siebten Brief« (142) des Baron von $\mathrm{F}^{* * *}$ integriert, vgl. zum philosophischen und ästhetischen Gehalt der Novelle Hans Richard Brittnacher: Dunkelmänner im Licht, S. 182f.

26 Ebd., S. 174. 
als »Konduktor« (75) die im Halbkreis Versammelten trifft, alles unterstützt vom dicken, optische Täuschungen befördernden Rauch, einem Komplizen im Kamin, ablenkenden Arrangements u.v.a.m. Kurz, die übernatürlichen Phänomene werden erfolgreich auf naturwissenschaftliche Gesetze, Taschenspielertricks und betrügerische Verabredungen reduziert. Allein der inkommensurable Rest einer selbst den Sizilianer überraschenden "zweiten Erscheinung" (76) des Armeniers irritiert noch den Prinzen.

Symptomatisch für die hier aufklärerisch-rationale Verfassung des Prinzen im ersten Buch des Geistersehers ist diese Schließ- und Schlüsselszene: Wenn der "Schließer« (92) die Zelle, in der der Sizilianer im Gefängnis einsitzt, nach dessen erhellender, dann alles erneut verrätselnder Binnen-Ich-Erzählung wieder verschließt, startet eine Aufklärungsmaschinerie ersten Ranges, in der nicht nur vom "Hörensagen" her geschlossen wird, sondern die den Sizilianer als "Augenzeuge«, der doch »keinen Zweifel« (79) am »Unergründlichen« (77) des Armeniers lassen wollte, restlos demontiert. Wenn hier buchstäblich dem Prinzen und dem Grafen von $\mathrm{O}^{* *}$ »ein plötzliches Licht [auf] ging“ (95), demonstriert das motivisch beinahe überdeutlich den aufklärerischen Gestus der Szene. Zentral wird bei dieser Ermittlungsszene ein Fehler, der dem Sizilianer in der bildreichen Geschichte seiner Erstbegegnung mit dem Armenier unterlaufen ist und der dem Prinzen im ermittelnden Nachgang dieser Vorgeschichte so anstößig wird, dass er die bisher ermittelten Erklärungen für je einzelne Phänomene nun (mit Recht) zum Verdacht auf eine Großverschwörung ausbaut. Den Anlass zu dieser verschwörungstheoretischen Gesinnungsänderung liefert einmal mehr ein Indiz: Der Ehering, den der Sizilianer im Dienste des verbrecherischen Lorenzo von $\mathrm{C}^{* * *}$ als effektheischendes Finale der inszenierten Trugbilder und als manifesten Beweis seines spirituellen Kontakts zum verschollenen Bruder Lorenzos erscheinen lässt und den dieser doch "gewiss nie vom Finger ließ « (94), überführt Lorenzo als Erbschleicher und Brudermörder sowie den Sizilianer als geldgierigen Komplizen der Erbschaftsintrige. Die ungelenken Ausflüchte des ansonsten so geständigen Sizilianers erregen beim Prinzen nun den Verdacht, man »habe sich des gröbern Gaukelspiels bedient, um dem feinern eine Folie unterzulegen" (98). Und das Feinere wäre, den "verwickelten Anschlag« als nur einen weiteren Teil der Machenschaften des Armeniers zu interpretieren, der kaum durch "Zufall«, sondern in "auffallender Harmonie" dieselbe "Person, zu derselben Zeit und an demselben Ort» (98) attackiere. Der Sizilianer, so der naheliegende Schluss des Prinzen, »war die Puppe, mit der er mich spielen läßt, während daß er selbst, unbeobachtet und unverdächtig, mit unsichtbaren Seilen mich umwindet« (98). 
Bemerkenswert ist bei dieser inneren Bewegung zudem die äußere, szenische Anordnung: Im abgeschlossenen Raum der Zelle des Sizilianers, die der "Schließer« (92) öffnet, um den wissensdurstigen Prinzen einzulassen, sind Denkschlüsse offenbar detailierbar und in dieser Kleinteiligkeit eingehegt. In dem Moment, wo der Prinz diese Zelle wieder verlässt, öffnet sich der Raum für den spekulierenden Überschuss - zunächst noch luzide vorangetrieben und aufschlussreich, dann aber jede Stoppregel missachtend gerät der Prinz in den Strom zweifelnder Eskalation.

Noch innerhalb der argumentativen Schließszene findet sich motivisch und metaphorisch nicht von ungefähr die materiale Schlüsselszene, nämlich der oben erwähnte indizierte Gang des verloren geglaubten Schatullenschlüssels, der beim Lotteriespiel als wunderbarer Gewinn wieder auftaucht. Die indexikalische Verschränkung aus Zeichenvollzug (deuten auf) und Zeichendeutung (etwas deuten) potenziert sich am Phänomen und Begriff des Schlüssels, der sowohl als motivischer Gegenstand (hier der verschwundene, dann epiphan wieder erscheinende Schlüssel) wie auch metaphorisch als Aufschluss gebend über die »wunderbaren Zufälle» (101) zunächst für verfügbar gehalten wird. Die Dichte, mit der in Schillers Text "Schlüsse" gezogen, jenes "Märchen aufgeschlüsselt« (100), »befriedigender Aufschluss gegeben« (75), ein "natürlicher Schlüssel« oder gar "zwei Schlüssel auf einmal« (102) zu einer rätselhaften Begebenheit gefunden werden, um nur einige Beispiele zu nennen, ist auffallend hoch:

Er [der Armenier] konnte davon erwarten, dass ich, früher oder später, aus eigenem Misstrauen oder fremdem Antrieb, den Schlüssel zu seinen Wundern in der Taschenspielerkunst aufsuchen würde. - Was konnte er Bessres tun, als daß er sie selbst nebeneinander stellte. (99)

Hier wird das Begriffsfeld schlie/sen einerseits zentral als Instrument natürlicher Erzähllogik markiert, gleichzeitig wird aber der bereits brüchig gewordenen Boden seines Sinns unterminiert, indem das unaufhörlich fortgesetzte Schließen in die asymptotische Spirale des Verdachts führt. Diese Brüchigkeit rührt nicht zuletzt von jener »Prophezeiung auf dem Markusplatz« (101) her, die den Tod des eigentlichen Thronfolgers auf die Stunde genau voraussagte und den Beginn aller venezianischen Verwicklungen des Prinzen mit dem Armenier bildet:Jenes »erste Wunder, welches alle übrigen eröffnet hat, bleibt nichts desto weniger ungeklärt; und was hilft uns der Schlüssel zu allen übrigen, wenn wir an der Auflösung dieses einzigen verzweifeln?« (101). Die dem Prinzen damit weiterhin notwendig scheinende Ermittlung der Bedingung der Möglichkeit einer solch erstaunlichen Voraussage, die 
intradiegetisch unergründbar bleibt, befeuert darum die Dynamik einer alles prinzipiell hinterfragenden, ja verdächtigenden Konspirationslogik. Hier wird Wahrscheinlichkeit (qua Indizien), also das asymptotische Verhältnis zwischen Tatsache und Deutungshoheit, als endloses Ermitteln zum entscheidenden Erzählparadigma des Romans.

Denn der Gedankenschluss als Sinnzuschreibung, das verknüpfende Schlussverfahren zwischen Tat und denkbarem Motiv als Verhältnismäßigkeit fallen scheinbar zunehmend aus. Ließen sich Dose, Ring, Geist und Schlüssel noch über naturwissenschaftliche Erklärungen oder intrigante Absprachen und Taschenspielertricks erklären, muss der Prinz nun einräumen:

Aber ich gebe Ihnen zu, daß meine Mutmaßung gekünstelt ist; ich gestehe, daß sie mich selbst nicht befriedigt. Ich bestehe nicht darauf [...], einen künstlichen und überlegten Entwurf zu Hülfe zu nehmen, wo man mit dem blo/sen Zufall schon ausreicht[.] (103; Hervorh. A.E.)

Der Prinz erweitert in dieser ermittelnden Szene (und damit um 1800 rechtshistorisch adäquat) das Indizienparadigma um sogenannte "gegenläufige Indizien ${ }^{27}$ wenn er zunächst über das Indiz »Ring« die Geschichte des Sizilianers eines Fehlschlusses überführt und darauf weitergehende Zweifel und den Verdacht einer omnipräsenten und -potenten Intrige des Armeniers hegt. Mit der finalen Volte aber, in der die zunächst noch für "natürlich« gehaltenen Schlüsse zu »künstlichen« umgewertet werden und damit aus der stabilisierenden Logik des Kausalnexus herausgelöst und paradigmatisch dem Verdacht der Künstlichkeit, dem »bloßen Zufall« einer Setzung ausgesetzt werden, öffnet sich das Feld der Willkürlichkeit von Zeichen und damit auch der auf sie bauenden Schlüsse. Fortan stürzen mit Einsatz des "zweiten Buchs" (104) der Prinz und dessen bis dahin von einer luziden Vernunft indizierten Denkschlüsse in eine Krise der Legitimation, da aufgrund der Zufälligkeit der verdächtigen Verweise keine Stoppregel vor Fehl- und Überinterpretationen schützt; so gerät der Prinz in eine halt- und heillose Spirale des abgründigen Zweifelns.

27 Paul Johann Anselm von Feuerbach: Lehrbuch des gemeinen in Deutschland gültigen Rechts, Gießen 1801, §544ff. 


\section{III.2 Das Indiziensterben}

Erst mit der Zulässigkeit "gegenläufiger u und nicht mehr nur "gleichgerichteter ${ }^{28}$ Indizien wird um 1800 rechtsgeschichtlich die Ambivalenz möglich: Zweideutigkeit entsteht dort, wo erst durch Zuschreibung von Qualitäten oder narrative Einbettung Sinnzusammenhänge einerseits konstruiert werden, anderseits evident scheinen, nie aber eineindeutig sind. Aus dieser Uneindeutigkeit erwachsen auch dem Prinzen Zweifel, die zudem von einer hereditär erworbenen und über seine Erziehung nur noch verstärkten religiösen Melancholie grundiert werden. Diese konstitutive und situative Konstellation gerät dem Prinzen zum Zweifel an allem, auch zum Zweifel am Prinzip des konstitutiven Zweifelns und so versinkt er in "Zweifelsucht«: "Ein entlarvter Betrug machte ihm auch die Wahrheit verdächtig, weil er sich die Wahrheit unglücklicherweise durch gleich schlechte Gründe bewiesen hatte« (106). Als Gegenmittel greift er zum Gefährlichsten, das das 18. Jahrhundert zu bieten hat: die "modernste Lektüre«. Durch eine "schlimme Hand « (107) bei der Auswahl handelt er sich durch überspitzte Rhetorik und feingesponnene Sophistik die vollständige Verwirrung seiner "Begriffe» (107) ein, bestärkt durch einen "Lieblingshang" zu »allem, was nicht begriffen werden soll« (108). Konsequent entsagt er dem konstruktiven Skeptizismus, tauscht "Vernunft « und "Herz« gegen das "»Labyrinth" des "glaubensreichen Schwärmers" und wird zum "ausgemachten Freigeist" (108). Schwärmerei, "Leidenschaft" und enthusiasmierte "Imagination" (108) bestimmen ihn fortan und bereiten den Boden für die "Libertinage« der "Sitten« (108) und damit die nächste indizierte (Liebes-)Dramaturgie des Armeniers, bestehend aus zu dechiffrierenden Zeichen und »feinen Trugschlüssen", die der Prinz "nicht lösen" (109) können wird.

Statt eine Kombination aus Ratio und Gefühl, Erfahrungswissen und Intuition, und damit eine zielführende (Ermittlungs-)Praxis im Umgang mit Indizien zu etablieren, oszilliert der Prinz im Gang der Geschichte stets zwischen den Extremen: totale Form oder totaler Stoff, ein Drittes gibt es für ihn nicht. Derjenige, der zu kombinieren, entelechisch indizierend zu agieren und zu manipulieren weiß, ist hingegen der Armenier. Darüber hinaus, so werde ich abschließend zeigen, folgt auch der Roman selbst der asymptotischen Form des indizierenden Narrativs.

28 Ebd. 


\section{III.3 Der Armenier: indizierte Entelechie}

Die Strategien des Armeniers folgen alle einer Keimzellenlogik, aus der heraus sich das Angelegte entwickeln und bei adäquater Zurichtung zum geplanten Zeitpunkt in der gewünschten Form zur Blüte gelangen soll, die der Armenier dieser Entwicklung zugedacht hat. Dass oft genug dieser selbst letzthin auch den Keim stiftet, dessen Früchte er final zu ernten beabsichtigt, markiert sein Vorgehen als eine Art indizierter Entelechie. Unverzichtbarer Teil dieser Indiziendramaturgie ist, dass die Vervollkommnung des jeweils Angelegten, zumindest dem Schein nach, sSelbstverwirklichung sein muss, d.h. sie muss als raus sich selbst heraus entwickelt $<$ wirken, obwohl sie genuin auf den Armenier zurückgeht. Allein die entelechische Illusion garantiert eine Überzeugungskraft, die keine Überredung, kein Liebesideal, kein finanzieller Ruin erzeugen kann und bildet die Grundstrategie dieses »unergründlichen« (77) Intriganten zwecks Täuschung und Manipulation des Prinzen.

Der Roman legt diese Lesart sehr nahe, animiert also die Lesenden zur durchaus spekulativen Lektüre dieser prospektiv manipulativen Zeichenstrategie: Über den Austausch des plötzlich verschwundenen Jägers durch Biondello gewinnt der Armenier die Informations- und Sozialkontrolle (Briefe werden abgefangen, Kontakte gestört oder lanciert etc.): Civitella stellt finanzielle und emotionale Abhängigkeiten her, die durch die Spielsucht initiiert und durch die Inszenierung der Episode mit der Griechin und die dem Prinzen schmeichelnde Aufnahme in die Geheimgesellschaft "Bucentauro« (108) befeuert werden. Die so ausgeübte ökonomische, emotionale und erotische Kontrolle über den Prinzen treibt ihn dem Kardinal als Hauptgläubiger und Mitglied der Kurie und damit der katholischen Kirche in die Arme. Letztere verbindet sich symptomatischerweise mit der Erotik über die schöne Griechin, die als »fromme Schwärmerin« (159) noch auf dem eigenen Sterbebett (»Spuren von Vergiftung«, 159) ${ }^{29}$ den Prinzen zur Konversion bringen will.

Der Geisterseher erzählt in der Aufklärungsepisode um den Sizilianer zunächst eine Art Erfolgsgeschichte der Indizien - einerseits. Andererseits arbeitet die referenzielle Einbettung in eine entelechische Illusion und die restriktive Rahmung der eineindeutigen, d.h. durch einen absoluten Souverän (Armenier) als immer schon srichtig^ qualifizierten Denkschlüsse gegen de-

${ }^{29}$ Vgl. zu den indexikalischen Verhältnissen bei Phänomenen der Vergiftung und des Giftmordes den Beitrag von Stephanie Langer in diesem Band. 
ren konstitutive Heteronomie. Damit erscheint die Ermittlungspraxis eines narrativ erschlossenen Sinnzusammenhangs über Indizien als immer schon korrumpiert. Dass im Roman ganz zentral das Prinzip des 'Schließens unterminiert wird, lässt sich als metaleptische Kritik an der machiavellistischen Indiziendramaturgie des Armeniers lesen. Mit ihrer prospektiven Verschwörungslogik evoziert die Erzählung eine erhebliche Unbehaglichkeit in der Manifestation solch wirksam werdender Realitätsprozeduren: Das "Steuer der Gerechtigkeit" (104) reißt der Romantorso keineswegs mehr herum, sondern prolongiert den Verdacht als Modell über sein ausbleibendes Ende hinaus.

\section{Wiederauferstehung: Indizierendes Erzählen, indiziertes Ermitteln}

Der Denkschluss, in dem mittelbar auf und von Tatsachen geschlossen wird, bindet Indizien als Material an ein erzählbares, sinnstiftendes Geschehen. Dennoch - oder gerade darum - gilt der "Beweis durch Vernunftschlüsse", und dazu gehören die »Indicien«, bei seinen Gegnern als »der gefährlichste und trüglichste unter allen ${ }^{30}{ }^{30}$ Dass auch der indizierende Nexus nicht ohne Denkschluss zu haben ist, zeigt ebenso souverän wie manipulativ herausfordernd Schillers Geisterseher.

Funktion und Wirkung von Indizien werden am Text selbst vorgeführt, nicht zuletzt indem das zweite Buch mit der finalen Leerstelle endet, die als »unerhörte Geschichte" (160) zwar angekündigt, aber deren Ende nicht erzählt werden wird. Ob oder gar wie der Prinz von seinen politischen Lenkern zur verbrecherischen Thronübernahme verleitet wird, bleibt der Spekulationslust der Lesenden überantwortet und damit, ganz indizienlogisch, für alle schlüssigen Deutungszuschreibungen offen. Der Schlüssel, gegenständlich zunächst verloren, dann wandernd, verborgen und epiphan wiederkehrend, wird metaphorisch als unzuverlässige, da unendlich perpetuierbare Schließmöglichkeit im Verlauf des Textes zunehmend unterminiert und ist selbst (oder gerade) für die zentral Handelnden, Erzählenden und zuletzt die Lesenden final nicht mehr zugänglich - so endet denn ein übers andere Mal ein Brief des Baron mit dem lakonischen Cliffhanger: »Den Schluss ein andermal« (149).

${ }^{30}$ Gallus Aloys Kleinschrod: Grundzüge der Theorie von Beweisen in peinlichen Sachen, in: Archiv des Criminalrechts 4/3 (1802), S. 44-85, hier S. 62 u. 73. 
Als asymptotische Form der seriellen Fortsetzung formt Schiller auch in metapoetischer Hinsicht sein Romanprojekt: Es bleibt unvollendet - und kann, folgt man der These der asymptotischen Heteronomie von Indizien hier poetologisch, auch nicht befriedigend beendet werden. Was die vom Armenier indizierte Entelechie auf der Ebene des Dargestellten freisetzt, wird auf der Ebene der Darstellung vorgängig potenziert: "Die Fortsetzung« (158) der "unerhörten Geschichte« (160) bleibt letztlich ungehört. Dieses asymptotische Konzept des Erzählens hätte dem Prinzen vermutlich gefallen, moniert er doch im Finale des Philosophischen Gesprächs:

Worauf Sie und andere ihre Hoffnung gründen, eben das hat die meinigen umgestürzt - eben diese geahndete Vollkommenheit der Dinge. Wäre nicht alles so in sich beschlossen, säh ich auch nur einen einzigen verunstaltenden Splitter aus diesem schönen Kreise herausragen, so würde mir das die Unsterblichkeit beweisen. (181)

Diese Splitter nun muss man im Geisterseher nicht lange suchen: Die Brüche des Textes, beispielsweise die in Klammern erscheinenden, vorausgreifenden Einschübe, die den "Ausgang" (101) einer Teilgeschichte vorwegnehmen (spurloses Verschwinden des Sizilianers aus dem Gefängnis), oder manche vernachlässigte Motivierung (»Ich habe vergessen zu erzählen, dass der Prinz schon seit etlichen Tagen einen Jäger vermisst«, 70), sind deutlich der Form des Fortsetzungsromans und seiner sukzessiven Entstehung geschuldet. Doch die erzähllogischen Brüche ebenso wie das fragmentarische und verrätselte Nichtende oder die korrumpierte Glaubwürdigkeit von Erzählern und Figuren sowie die schwankende Position zwischen Allwissen und Nichtwissen, die die Lesenden in eine indizienlogische Pflicht zweiter Ordnung nimmt - all dies bildet semantisch und strukturell ab, was das Indiz poetologisch in seiner heteronomen und liminalen Potentialität als Bedingung vieldeutiger Möglichkeiten für ein Erzählen als Ermitteln bereitstellt: die "genetische Darstellung" von der (Un-)Wahrscheinlichkeit der Literatur. ${ }^{31}$

31 Friedrich Schiller: Werke und Briefe, Bd. 5, S. 1001. 
